\title{
14 Years after the Deepwater Horizon Spill: \\ 2 Molecular Transformation of Macondo Well Oil in 3 Louisiana Salt Marsh Sediments Revealed by FT- \\ 4 ICR Mass Spectrometry
}

5 Huan Chen, ${ }^{\dagger}$ Aixin Hou, ${ }^{\S}$ Yuri E. Corilo, ${ }^{\dagger}$ Qianxin Lin,,$"$ Jie Lu, ${ }^{\ddagger}$ Irving

6 A. Mendelssohn, "Rui Zhang, ${ }^{\S}$ Ryan P. Rodgers ${ }^{\dagger, \ddagger}$ and Amy M.

7 McKenna* ${ }^{*}$

$8{ }^{\dagger}$ National High Magnetic Field Laboratory and ${ }^{\ddagger}$ Future Fuels Institute, Florida State

9 University ,1800 East Paul Dirac Drive, Tallahassee, FL 32310, United States

10 §Department of Environmental Sciences, College of the Coast and Environment,

11 Louisiana State University, 1285 Energy, Coast, and Environment Building, Baton

12 Rouge, Louisiana 70803, United States

13 "Department of Oceanography and Coastal Sciences, College of the Coast and

14 Environment, Louisiana State University, 1002-Y Energy, Coast, and Environment

15 Building, Baton Rouge, Louisiana 70803, United States.

16 *To whom correspondence should be addressed. Tel.: +1 850644 4809; Fax +1 8506441366.

17 E-mail address: mckenna@magnet.fsu.edu

19 Number of pages: 22

20 Number of figures: 5

$21 \quad$ Number of tables: 3 
ם SUPPORTING INFORMATION

\section{Supporting Method Details}

Sample preparation. Macondo wellhead crude was provided by British Petroleum oil company through the Gulf Coast Restoration Organization. All solvents were HPLC grade (Sigma-Aldrich Chemical Co., St. Louis, MO). Prior to mass spectral analysis, $\sim 10$ $\mathrm{mg}$ of crude oil and sediment extracts were diluted in toluene to make a stock solution $(1 \mathrm{mg} / \mathrm{mL}$ ) that was further diluted to yield a final concentration of $250 \mu \mathrm{g} / \mathrm{mL}$ with equal parts (vol:vol) methanol spiked with $2 \%$ (by volume) formic acid (positive ESI) or $0.250 \%$ (by volume) tetramethylammonium hydroxide (TMAH, $25 \%$ by weight in methanol, negative ESI) to ensure efficient protonation/deprotonation prior to FT-ICR mass spectral analysis.

Instrumentation: ESI Source. Sample solutions were infused via a microelectrospray source $^{1}$ ( $50 \mu \mathrm{m}$ i.d. fused silica emitter) at $500 \mathrm{~nL} / \mathrm{min}$ by a syringe pump. Ions formed at atmospheric pressure enter through a dual-stage rf-focusing ion funnel interface ${ }^{2}$ (funnel 1: 2.7 Torr; funnel 2: 270 mTorr) with a voltage gradient transfer of ions into the mass spectrometer $(\mathrm{DC} 1=100 \mathrm{~V} ; \mathrm{DC}=70 \mathrm{~V} ; \mathrm{DC} 3=40 \mathrm{~V} ; \mathrm{DC} 4=10 \mathrm{~V})$.

Instrumentation: 9.4 T FT-ICR MS. Parent Macondo well oil (MWO) and oil extracts were analyzed with a custom-built FT-ICR mass spectrometer ${ }^{3}$ equipped with a $9.4 \mathrm{~T}$ horizontal $220 \mathrm{~mm}$ bore diameter superconducting solenoid magnet operated at room temperature, and modular ICR data station (Predator) ${ }^{4}$ facilitated instrument control, data acquisition, and data analysis. Ions generated at atmospheric pressure were accumulated in an external quadrupole (100-500 ms), passed through an rf-only quadrupole into an octopole equipped with titled wire extraction electrodes for improved ion extraction and transmission. ${ }^{5}$ Helium gas introduced into the octopole collisionally cools ions prior to transfer through rf-only quadrupoles (total length $127 \mathrm{~cm}$ ) equipped with an auxiliary if waveform ${ }^{6}$ into a 7 -segment open cylindrical cell ${ }^{7}$ based on the Tolmachev configuration. ${ }^{8} 100$ individual transients of $5.9 \mathrm{~s}$ duration were signal averaged. Data was collected at maximum memory depth of the data station hardware 
(16 million samples), apodized with a single-sided Hanning apodization, and zero-filled to 16 megasample (16777216 samples or $2^{24}$ ). An additional zero fill brings the preFT data packet to 32 megasample. Due to increased complexity at higher $\mathrm{m} / \mathrm{z}$, broadband phase correction $^{9,10}$ was applied to mass spectra for salt marsh extracts and parent MWO to increase the resolution of isobaric species. For all mass spectra, the achieved mass spectral resolving power approached the theoretical limit ${ }^{11}$ across the entire mass range, e.g., average resolving power, $\mathrm{m} / \Delta \mathrm{m}_{50 \%}$, in which $\Delta \mathrm{m}_{50 \%}$ is mass spectral peak width at half maximum peak height, was $\sim 1,000,000-1,200,000$ at $\mathrm{m} / \mathrm{z} 500$. All samples were analyzed in triplicate.

Mass Calibration and Data Analysis. ICR frequencies were converted to ion masses based on the quadrupolar trapping potential approximation. ${ }^{12,13}$ Each $\mathrm{m} / \mathrm{z}$ spectrum was internally calibrated with respect to an abundant homologous alkylation series whose by isotopic fine structure based on the "walking" calibration equation. ${ }^{14}$ Experimentally measured masses were converted from the International Union of Pure and Applied Chemistry (IUPAC) mass scale to the Kendrick mass scale ${ }^{15}$ for rapid identification of homologous series for each heteroatom class (i.e., species with the same $\mathrm{C}_{c} \mathrm{H}_{h} \mathrm{~N}_{n} \mathrm{O}_{o} \mathrm{~S}_{s}$ content, differing only by degree of alkylation). ${ }^{16}$ Peak assignments were performed by Kendrick mass defect analysis. ${ }^{16}$ For each elemental composition, $\mathrm{C}_{c} \mathrm{H}_{h} \mathrm{~N}_{n} \mathrm{O}_{o} \mathrm{~S}_{s}$, the heteroatom class, type (double bond equivalents, DBE = number of rings plus double bonds to carbon, $\mathrm{DBE}=\mathrm{C}-h / 2+n / 2+1)^{17}$ and carbon number, $c$, were tabulated for subsequent generation of heteroatom class relative abundance distributions and graphical relative abundance-weighted DBE vs. carbon number images by a custom software package (PREDATOR and PetroOrg, copyright 2012-2014).

Elemental analysis. Elemental analysis was performed with a Thermo Finnigan Elemental Analyzer (FLASH EA 112, San Jose, CA, USA) to determine CHNS (run 1) and $\mathrm{O}$ content (run 2) in two separate experiments. For CHNS analysis, 1.5-2.75 mg of sample was placed in a tin cup, crushed to form a sphere, and placed in the autosampler. All samples were analyzed in quadruplicate. Calibration of the instrument 
is provided by the analysis of standards of known elemental content, and all 85 quadruplicate runs included a separate, independent standard not used in the initial calibration. A similar procedure was followed for oxygen measurements, but with a silver cup. Lubricant reference material 0104 (Thermo) was used as the standard to calibrate the instrument and Lubricant oil 100608 (Microlab s.n.c.) was analyzed together with samples as an independent standard in each run. For $\mathrm{O}$ analysis, about 2 $\mathrm{mg}$ samples were placed in silver cups and measured by pyrolysis at $1060^{\circ} \mathrm{C}$. Standard materials 2, 5-bis-(5-tert-butylbenzoxazol-2-yl)-thiophene (BBOT) and sulfanilamide (C. E. Elantech, Inc.) were used for calibration.

GC $\times$ GC-TOFMS Analysis. Comprehensive two-dimensional gas chromatography coupled to a time-of-flight mass spectrometry (GC $\times$ GC-TOFMS) from LECO (St. Joseph, MI) was used for the identification of petroleum hydrocarbons. Samples were dissolved in dichloromethane (DCM) at $500 \mathrm{mg} \mathrm{mL}^{-1}$ and $1 \mu \mathrm{L}$ each was injected with 10:1 split. The first dimension column was a BP-1 (60 m x $0.25 \mathrm{~mm}$ ID, $0.25 \mu \mathrm{m}$ film thickness, $100 \%$ polysiloxane), and the second dimension column was a BPX50 (1.5 m x $0.1 \mathrm{~mm}$ ID, $0.1 \mu \mathrm{m}$ film thickness, 50\% Phenyl). Both columns were purchased from

SGE (Ringwood, VA, Australia). Helium was used as carrier gas with the flow rate of 1.5 $\mathrm{mL} \mathrm{min}^{-1}$. The $\mathrm{GC}$ inlet temperature was $300^{\circ} \mathrm{C}$. The $\mathrm{GC}$ oven temperature was initially held at $40{ }^{\circ} \mathrm{C}$ for $0.5 \mathrm{~min}$, then ramped at $5{ }^{\circ} \mathrm{C} \mathrm{min}{ }^{-1}$ to $340{ }^{\circ} \mathrm{C}$ and held for $10 \mathrm{~min}$. The secondary oven had a temperature offset of $+5{ }^{\circ} \mathrm{C}$ from the first oven. The modulator had an offset of $+10{ }^{\circ} \mathrm{C}$. The modulation period was $6 \mathrm{~s}$ with hot pulse of $0.8 \mathrm{~s}$. Electron-impact ionization at $70 \mathrm{eV}$ was used. The ion source was set at $200{ }^{\circ} \mathrm{C}$ and the transfer line at $280^{\circ} \mathrm{C}$. The MS data was collected with acquisition rate of $200 \mathrm{spectra} / \mathrm{s}$ and mass range from 40 to $500 \mathrm{~m} / \mathrm{z}$.

GC $\times$ GC -FID Analysis. GC $\times$ GC coupled to a flame ionization detector $(G C \times G C-$ FID) from LECO was used for the quantitation of petroleum biomarkers in MWO and select sediment extracts. Briefly, $1 \mu \mathrm{l}$ of each sample at $50 \mathrm{mg} \mathrm{mL}-1$ in DCM was injected splitless. The first and second dimensional columns were the same as for $\mathrm{GC} \times$ GC-TOFMS. The GC inlet temperature was $300^{\circ} \mathrm{C}$. The carrier gas was Helium with a 
115 flow rate of $1.00 \mathrm{~mL}$ min-1. The secondary oven had a temperature offset of $+5{ }^{\circ} \mathrm{C}$ from 116 the first oven and the modulator had an offset of $+10^{\circ} \mathrm{C}$. The modulation period was 10 $117 \mathrm{~s}$ with hot pulse of $1.5 \mathrm{~s}$. The FID temperature was set at $340^{\circ} \mathrm{C}$.

$\mathrm{C}_{30}$-hopane and other Biomarker compounds were identified and confirmed by $\mathrm{GC} \times$ GC-TOFMS, published elution order ${ }^{18,19}$ and the petroleum standard reference material (SRM) 2266 purchased from the National Institute of Standards and Technology (NIST). GC $\times$ GC-FID peaks were manually integrated using the ChromaTOF software (Leco, $V$ 4.50.8.0). NIST SRM 2266 was injected at five concentrations and a linear calibration curve was obtained for the certified biomarkers present $\left(R^{2}>0.999\right)$.

Calculation of Oil Loss. The depletion of oil in the filed samples was estimated by measuring the concentration of weathering-resistant $\mathrm{C}_{30}$-hopane relative to its concentration in the parent MWO according to eq $1^{20}$.

Total oil loss $(\%)=\left(1-\frac{H_{M W O}}{H_{W}}\right) \times 100$

Where the amount of oil degraded is estimated by the concentration of $\mathrm{C}_{30}$-hopane in the weathered oil sample $(\mathrm{Hw})$ relative to its concentration in the parent MWO $\left(\mathrm{H}_{\mathrm{MWO}}\right)$. Individual compound $\mathrm{X}$ depletion, normalized to C30-hopane is determined by eq $2^{21}$ $\mathrm{C}_{\mathrm{w}}$ is the compound $\mathrm{X}$ concentration in the weathered oil and $\mathrm{C}_{\mathrm{MWO}}$ is the concentration of compound $\mathrm{X}$ in the MWO. Concentration was calculated on an oil-weight basis by converting the peak volumes of compound in the GC $\times$ GC-FID chromatogram of a specific sample according to the standard curve of NIST SRM 2266.

Separation of Naphthenic Acids. Parent MWO and oil residues collected at 9, 36 and 48 mos post-spill were further subjected to amino-propyl silica extraction (APS) to selectively isolate naphthenic acids as previously reported. ${ }^{22}$ APS separation exploits 
145 the strong hydrogen bonding interaction between APS and carboxylic acids and 146 subsequently allows the elution of acids in the oil samples by displacing them with 147 formic acid. Briefly, samples were weighed, dissolved in $1 \mathrm{~mL}$ dichloromethane, and 148 loaded onto pre-conditioned (with dichloromethane) solid phase extraction (SPE) cartridge (Agilent Bond Elut $\mathrm{NH}_{2} 2 \mathrm{~g}$ ). After equilibration, non-acidic (fraction 1) were eluted with $100 \%$ (by volume) dichloromethane, and moderately acidic (fraction 2 ) were sequentially eluted with 50/50 (v/v) dichloromethane:methanol. Carboxylic acids (fraction 3) were eluted with 47.5/47.5/5 (v/v/v) dichloromethane:methanol:formic acid by displacement from the stationary phase that occurs with the addition of formic acid. ${ }^{22}$ All three fractions were desolvated under dry nitrogen gas and weighed. Percentage mass recovery was calculated by dividing the mass recovered from each fraction by the total sample mass.

Basic Speciation by Positive-ion Electrospray lonization. Positive-ion ESI of 159 petroleum selectively ionizes pyridinic nitrogen, the strongest bases in crude oil, and the most recalcitrant nitrogen compounds, ${ }^{23-25}$ due to low reactivity. ${ }^{26}$ Figure S-5 shows the heteroatom class distribution for basic species in MWO and sediment extracts, with pyridinic (6-membered ring) nitrogen $\left(\mathrm{N}_{1}\right)$ as the most abundant class in MWO and all extracts, followed by $\mathrm{N}_{1} \mathrm{O}_{1}, \mathrm{O}_{1}$ and $\mathrm{N}_{1} \mathrm{O}_{2}$ classes. Across all time points, there is very little change in the relative abundance of basic species detected by positive-ion ESI FTICR MS, consistent with the known recalcitrance of pyridinic nitrogen-containing compounds in the environment and oil upgrading processes. Oxygen-containing compounds $\left(\mathrm{O}_{1}\right.$ and $\left.\mathrm{O}_{2}\right)$ were also observed in positive-ESI across all samples, in agreement with previous reports of transformation products in Macondo oil residues. ${ }^{27}$

\section{References:}

1. Emmett, M. R.; White, F. M.; Hendrickson, C. L.; Shi, S. D.-H.; Marshall, A. G., Application of micro-electrospray liquid chromatography techniques to FT-ICR MS to enable high-sensitivity biological analysis. J. Am. Soc. Mass Spectrom. 1998, 9, (4), 333-340. 
3. Kaiser, N. K.; Quinn, J. P.; Blakney, G. T.; Hendrickson, C. L.; Marshall, A. G., A Novel 9.4 Tesla FTICR Mass Spectrometer with Improved Sensitivity, Mass Resolution, and Mass Range. J. Am. Soc. Mass Spectrom. 2011, 22, (8), 1343-1351.

4. Blakney, G. T.; Hendrickson, C. L.; Marshall, A. G., Predator data station: A fast data acquisition system for advanced FT-ICR MS experiments. Int. J. Mass Spectrom. 2011, 306, (23), 246-252.

5. Wilcox, B. E.; Hendrickson, C. L.; Marshall, A. G., Improved ion extraction from a linear octopole ion trap: SIMION analysis and experimental demonstration. J. Am. Soc. Mass Spectrom. 2002, 13, 1304-1312.

6. Kaiser, N. K.; Savory, J. J.; Hendrickson, C. L., Controlled ion ejection from an external trap for extended $m / z$ range in FT-ICR mass spectrometry. J. Am. Soc. Mass Spectrom. 2014, 25, 943-949.

7. Kaiser, N. K.; Savory, J. J.; McKenna, A. M.; Quinn, J. P.; Hendrickson, C. L.; Marshall, A. G., Electrically compensated Fourier transform ion cyclotron resonance cell for complex mixture mass analysis. Anal. Chem. 2011, 83, (17), 6907-6910.

8. Tolmachev, A. V.; Robinson, E. W.; Wu, S.; Kang, H.; Lourette, N. M.; Pasa-Tolic, L.; Smith, R. D., Trapped-Ion Cell with Improved DC Potential Harmonicity for FT-ICR MS. J. Am. Soc. Mass Spectrom. 2008, 19, (4), 586-597.

9. Xian, F.; Hendrickson, C. L.; Blakney, G. T.; Beu, S. C.; Marshall, A. G. In Broadband Phase Correction of Complex FT-ICR Mass Spectra, 1-5 June 2008, Denver, CO, 2008; Proc. 56th ASMS Conf. on Mass Spectrometry \& Allied Topics: Denver, CO, 2008; p TP008.

10. Xian, F.; Hendrickson, C. L.; Blakney, G. T.; Beu, S. C.; Marshall, A. G., Automated Broadband Phase Correction of Fourier Transform Ion Cyclotron Resonance Mass Spectra. Anal. Chem. 2010, 82, (21), 8807-8812.

11. Marshall, A. G.; Hendrickson, C. L.; Jackson, G. S., Fourier transform ion cyclotron mass spectrometry: A primer. Mass Spectrom. Rev. 1998, 17, (1), 1-35.

12. Shi, S. D. H.; Drader, J. J.; Freitas, M. A.; Hendrickson, C. L.; Marshall, A. G., Comparison and interconversion of the two most common frequency-to-mass calibration functions for Fourier transform ion cyclotron resonance mass spectrometry. Int. J. Mass Spectrom. 2000, 195, 591598.

13. Grosshans, P. B.; Shields, P. J.; Marshall, A. G., Comprehensive Theory of the Fourier Transform Ion Cyclotron Resonance Signal for All Ion Trap Geometries. J. Chem. Phys. 1991, 94, 5341-5352.

14. Savory, J. J.; Kaiser, N. K.; McKenna, A. M.; Xian, F.; Blakney, G. T.; Rodgers, R. P.; Hendrickson, C. L.; Marshall, A. G., Parts-Per-Billion Fourier Transform lon Cyclotron Resonance Mass Measurement Accuracy with a "Walking" Calibration Equation. Anal. Chem. 2011, 83, (5), 1732-1736.

15. Kendrick, E., A Mass Scale Based on $\mathrm{CH}_{2}=14.0000$ for High Resolution Mass Spectrometry of Organic Compounds. Anal. Chem. 1963, 35, (13), 2146-2154.

16. Hughey, C. A.; Hendrickson, C. L.; Rodgers, R. P.; Marshall, A. G.; Qian, K., Kendrick Mass Defect Spectroscopy: A Compact Visual Analysis for Ultrahigh-Resolution Broadband Mass Spectra. Anal. Chem. 2001, 73, 4676-4681.

17. McLafferty, F. W.; Turecek, F., Interpretation of Mass Spectra. 4th ed. ed.; University Science Books: Mill Valley, CA, 1993; p 371.

18. Gaines, R. B.; Frysinger, G. S.; Hendrick-Smith, M. S.; Stuart, J. D., Oil spill source identification by comprehensive two-dimensional gas chromatography. Environ. Sci. Technol. 1999, 33, (12), 2106-2112.

19. Eiserbeck, C.; Nelson, R. K.; Grice, K.; Curiale, J.; Reddy, C. M., Comparison of GC-MS, GC-MRM-MS, and GC $\times$ GC to characterise higher plant biomarkers in Tertiary oils and rock extracts. Geochim. Cosmochim. Acta 2012, 87, 299-322. 
20. Douglas, G. S.; Bence, A. E.; Prince, R. C.; McMillen, S. J.; Butler, E. L., Environmental stability of selected petroleum hydrocarbon source and weathering ratios. Environ. Sci. Technol. 1996, 30, (7), 2332-2339.

21. Aeppli, C.; Nelson, R. K.; Radović, J. R.; Carmichael, C. A.; Valentine, D. L.; Reddy, C. M., Recalcitrance and degradation of petroleum biomarkers upon abiotic and biotic natural weathering of Deepwater Horizon oil. Environ. Sci. Technol. 2014, 48, (12), 6726-6734. 22. Rowland, S. M.; Robbins, W. K.; Corilo, Y. E.; Marshall, A. G.; Rodgers, R. P., Solid-Phase Extraction Fractionation To Extend the Characterization of Naphthenic Acids in Crude Oil by Electrospray Ionization Fourier Transform Ion Cyclotron Resonance Mass Spectrometry. Energy Fuels 2014, 28, (8), 5043-5048.

23. Zeuthen, P.; Knudsen, K. G.; Whitehurst, D. D., Organic nitrogen compounds in gas oil blends, their hydrotreated products and the importance to hydrotreatment. Catal. Today 2001, 65, 307-314.

24. Zhang, H.; Li, G.; Jia, Y.; Liu, H., Adsorptive removal of nitrogen-containing compounds from fuel. J. Chem. Eng. Data 2010, 55, (1), 173-177.

25. Wiwel, P.; Hinnemann, B.; Hidalgo-Vivas, A.; Zeuthen, P.; Petersen, B. O.; Duus, J. O., Characterization and identification of the most refractory nitrogen compounds in hydroprocessed vacuum gas oil. Ind. Eng. Chem. 2010, 49, (7), 3184-3193.

26. Wiwel, P.; Knudsen, K.; Zeuthen, P.; Whitehurst, D., Assessing compositional changes of nitroge compounds during hydrotreating of typical diesel range gas oils using a novel preconcentration technique coupled with gas chromatography and atomic emission detection. Ind. Eng. Chem. Res. 2000, 39, (2), 533-540.

27. Ruddy, B. M.; Huettel, M.; Kostka, J. E.; Lobodin, V. V.; Bythell, B. J.; McKenna, A. M.; Aeppli, C.; Reddy, C. M.; Nelson, R. K.; Marshall, A. G., Targeted petroleomics: analytical investigation of Macondo well oil oxidation products from Pensacola Beach. Energy Fuels 2014, 28, (6), 4043-4050. 


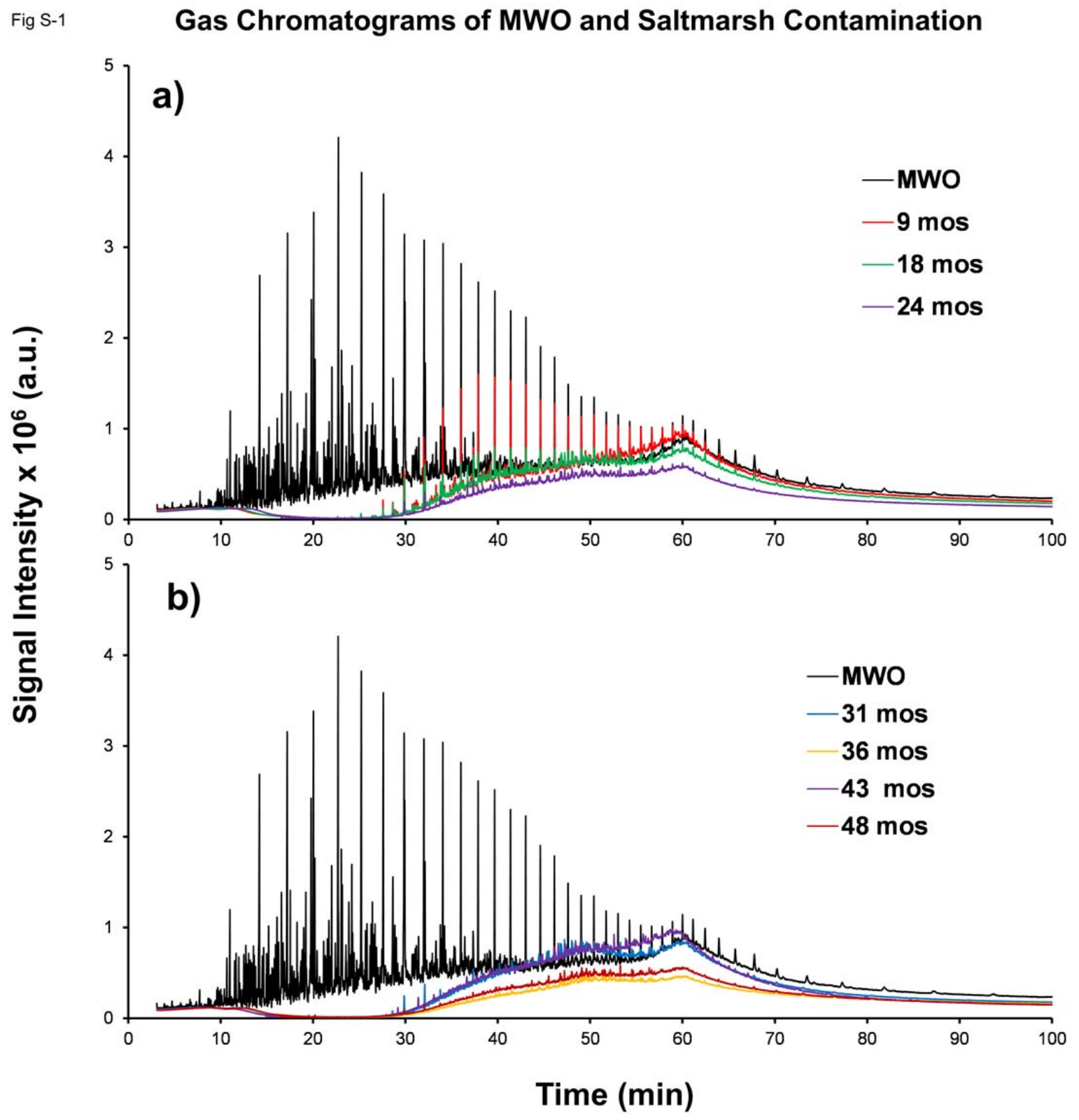

Figure S1. Overlaid GC chromatograms of MWO and saltmarsh extracts collected at heavily 259 oiled saltmarsh sites from a) 9, 18 and 24 mos and b) 31, 36, 43 and 48 mos. In the first 9 mos, 260 the complete loss of low molecular weight aromatic hydrocarbons and normal alkanes (below $261 \sim n \mathrm{C}_{20}$ ) through evaporation, water-washing and other weathering processes occurred relative to 262 the MWO. 
Figure S-2
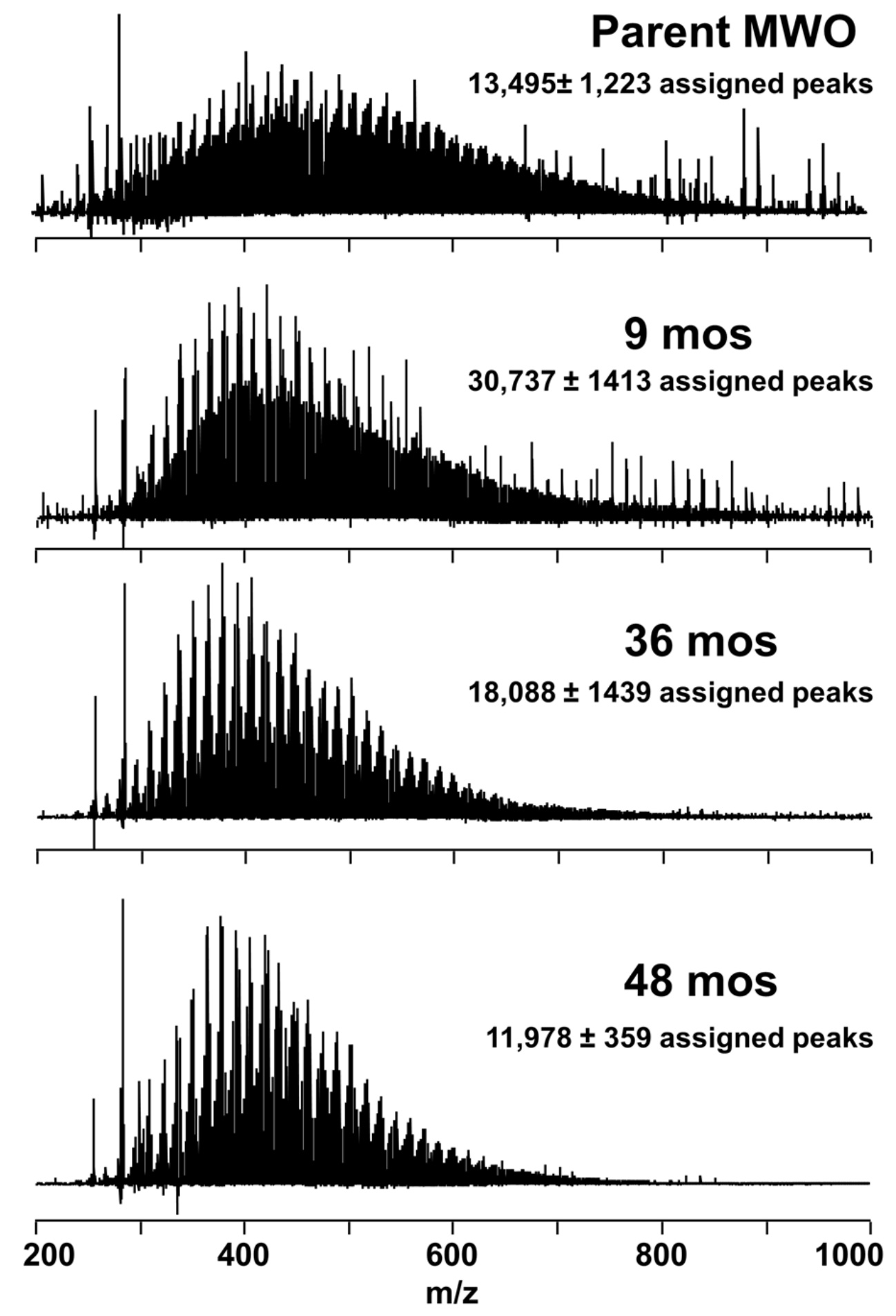

Figure S-2. Negative ion electrospray ionization (ESI) FT-ICR spectra for parent MWO and the weathered sediment collected 9 mos, 36 mos, 48 mos and mass spectra peak numbers. Mass spectra are normalized to the largest peak in each sample. Average errors based on

267 triplicate analysis. 


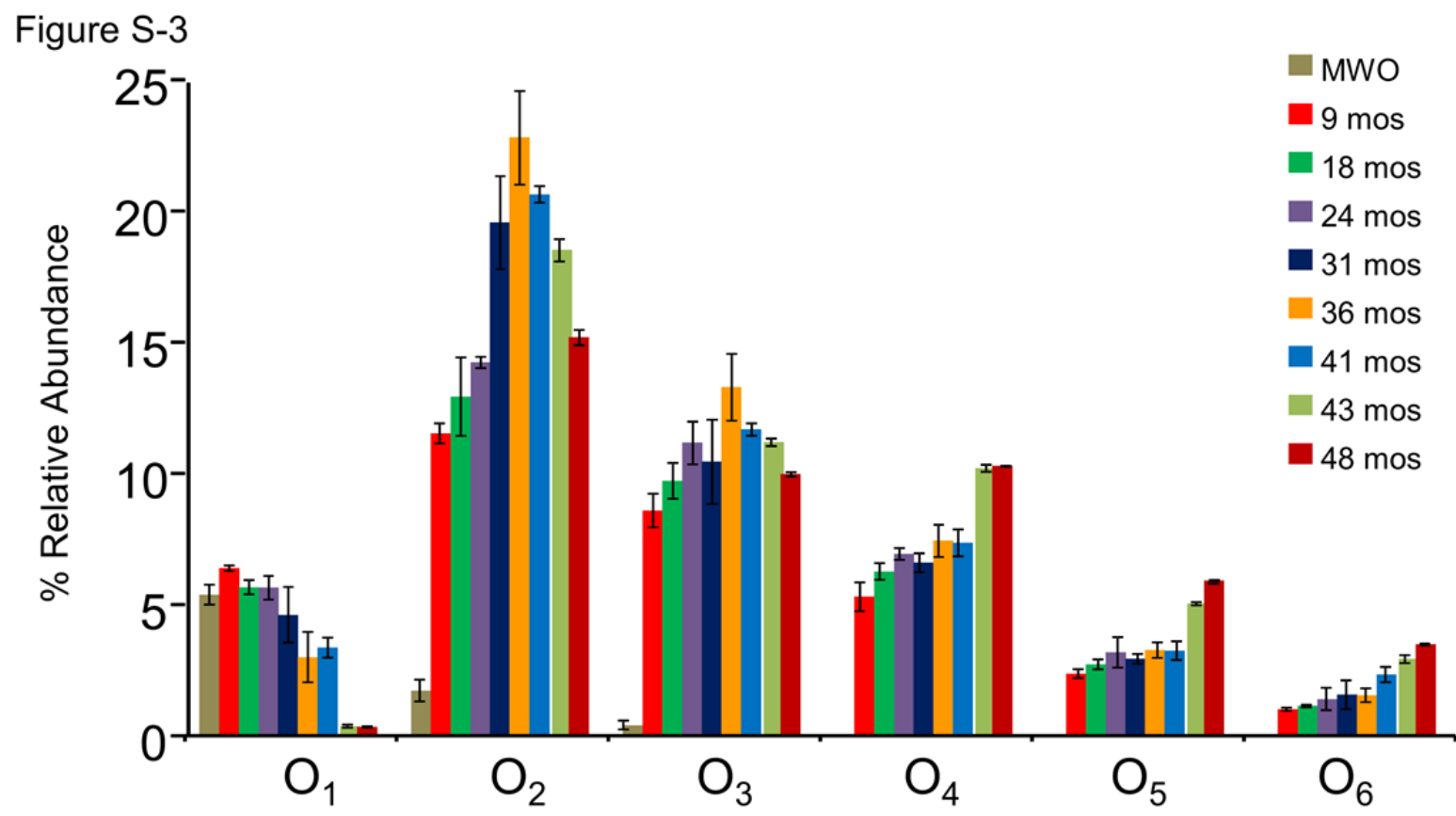

269 Figure S-3. Expanded view of $\mathrm{O}_{x}$ heteroatom classes derived from negative ESI FT-ICR mass 270 spectra of parent MWO Macondo well oil and oil contaminants over 48 mos post-spill with a 271 TMAH-modified solvent system. 
Figure S-4

Acidic Speciation

Heteroatom Class Distribution

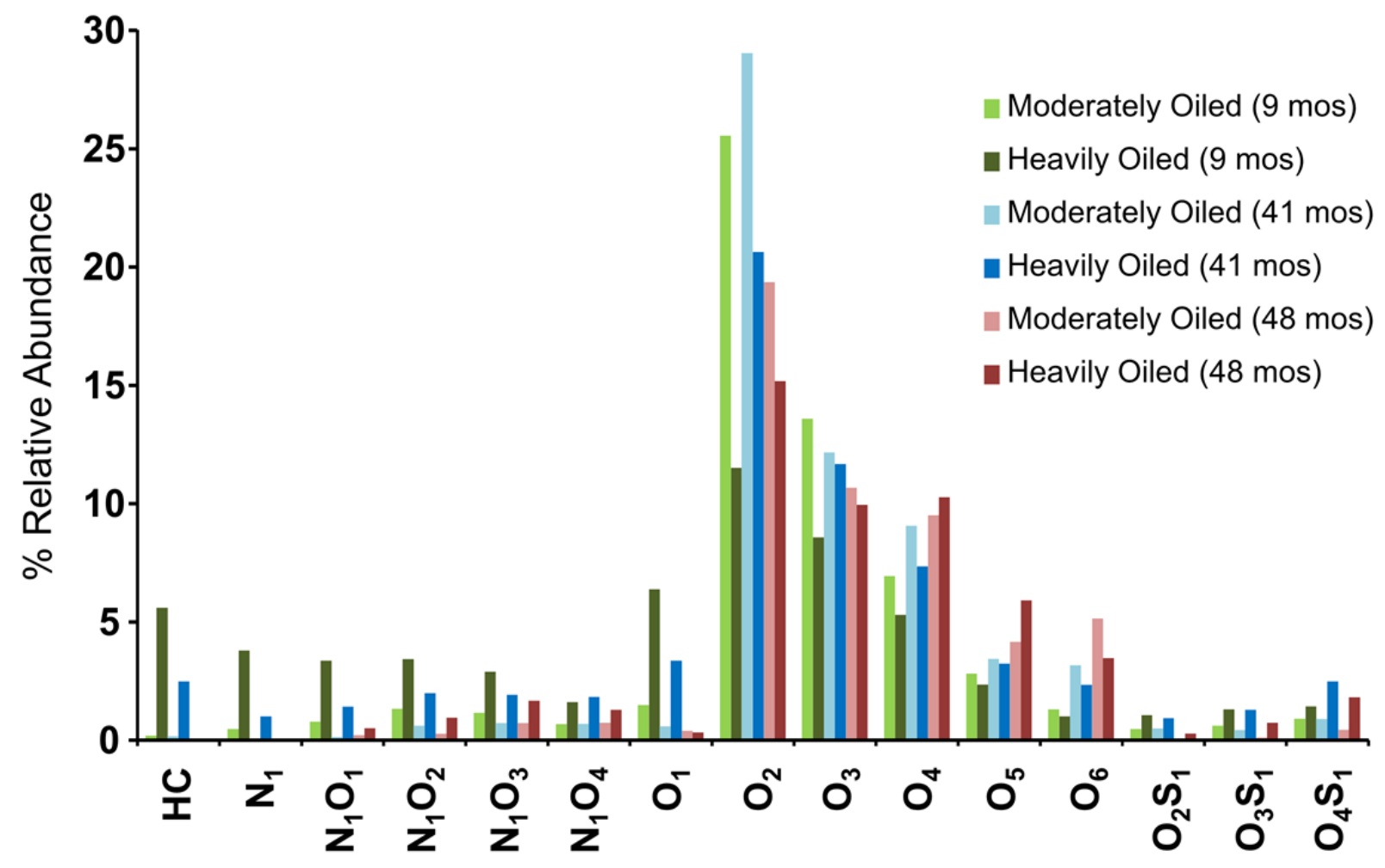

273

274

275

276

Figure S-4. Heteroatom class distribution of select acidic species (>1\% relative abundance) analyzed by negative ion ESI FT-ICR MS for the field samples collected from heavily oiled sites and moderately oiled sites, 9 mos, 41 and 48 mos post the oil spill. 


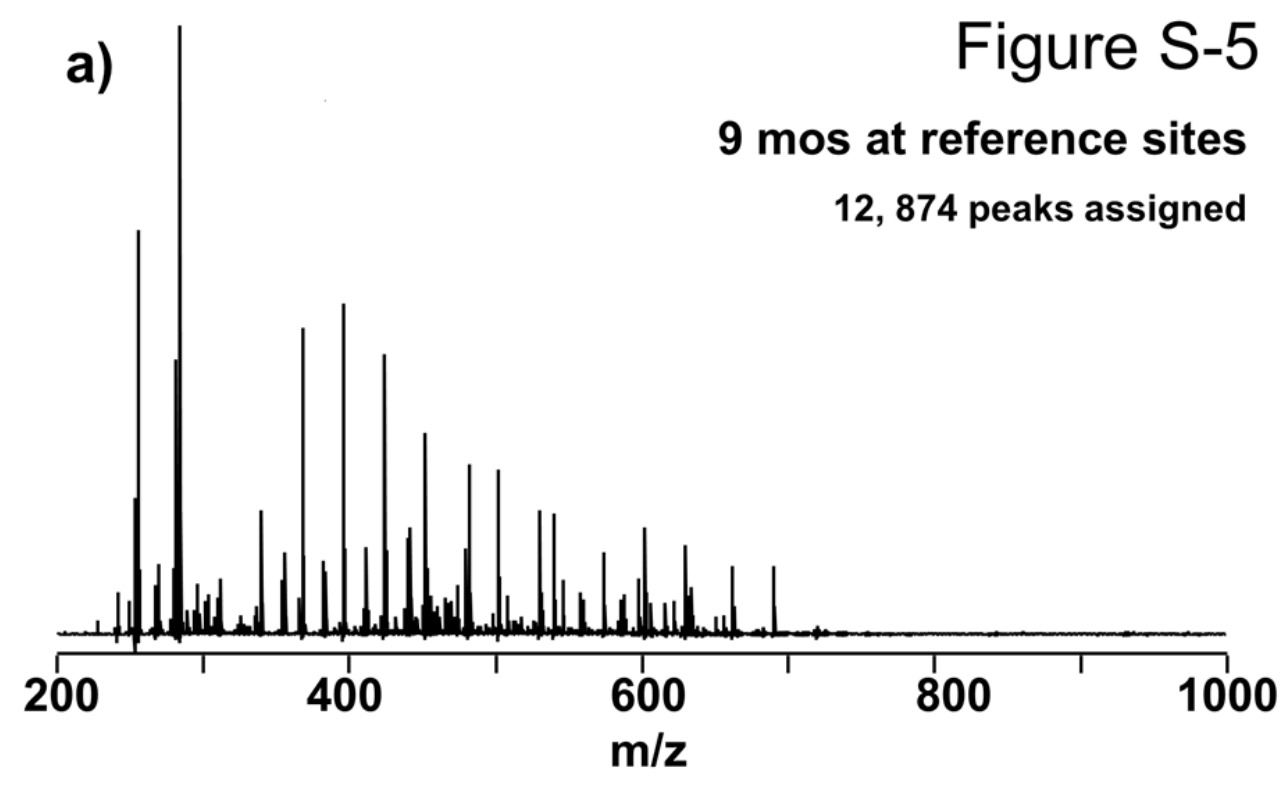

b)

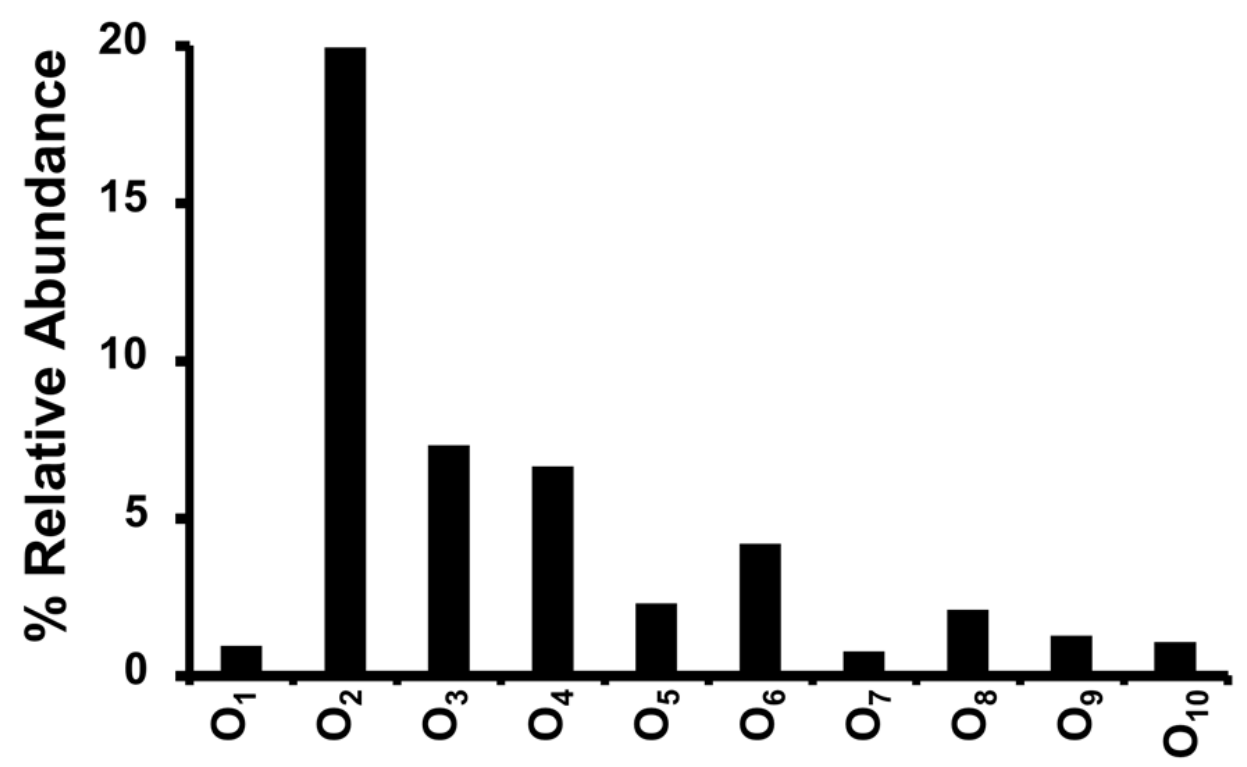

Heteroatom Class

278 Figure S-5. Negative ion electrospray ionization (ESI) FT-ICR spectrum (a) and heteroatom 279 class distribution (b) for sediment samples collected from the reference sites 9 mos post spill. 
282 Table S-1. Sampling time points and degree of contamination for salt marsh samples

283 collected from Barataria Bay, Louisiana from January 2011 to April 2014.

\begin{tabular}{|c|c|c|c|c|}
\hline $\begin{array}{l}\text { Sampling } \\
\text { Date }\end{array}$ & $\begin{array}{c}\text { Degree of Oiling } \\
\text { (SCAT) }\end{array}$ & $\begin{array}{l}\text { Homogenized } \\
\text { Sites }\end{array}$ & $\begin{array}{l}\text { Post-Spill } \\
\text { (Mos) }\end{array}$ & $\begin{array}{l}\text { (g) Extracted / } 20 \\
\text { g sediment }\end{array}$ \\
\hline Jan. 2011 & Heavily Oiled & $1-7$ & 9 & 5.199 \\
\hline Oct. 2011 & Heavily Oiled & $1-7$ & 18 & 2.008 \\
\hline Apr. 2012 & Heavily Oiled & $1-7$ & 24 & 1.331 \\
\hline Nov. 2012 & Heavily Oiled & $1-7$ & 31 & 1.294 \\
\hline Apr. 2013 & Heavily Oiled & $1-7$ & 36 & 0.579 \\
\hline Sep. 2013 & Heavily Oiled & $1-7$ & 41 & 0.456 \\
\hline Nov. 2013 & Heavily Oiled & $1-7$ & 43 & 0.512 \\
\hline Apr. 2014 & Heavily Oiled & $1-7$ & 48 & 0.482 \\
\hline Jan. 2011 & Moderately Oiled & $8-14$ & 9 & 0.470 \\
\hline Sep. 2013 & Moderately Oiled & $8-14$ & 41 & 0.0647 \\
\hline Apr. 2014 & Moderately Oiled & $8-14$ & 48 & 0.0119 \\
\hline Jan. 2011 & Reference Sites & $15-21$ & 9 & 0.0015 \\
\hline Sep. 2013 & Reference Sites & $15-21$ & 41 & 0.0012 \\
\hline Apr. 2014 & Reference Sites & $15-21$ & 48 & 0.0011 \\
\hline
\end{tabular}


Table S-2. Elemental analysis of parent MWO and sediment extracts from heavily contaminated sites.

\begin{tabular}{|c|c|c|c|c|c|c|c|c|c|}
\hline & $\begin{array}{c}\text { Parent } \\
\text { MWO }\end{array}$ & 9 mos & 18 mos & 24 mos & 31 mos & 36 mos & 41 mos & 43 mos & 48 mos \\
\hline Carbon & $85.9^{\mathrm{a}} \pm 0.01^{\mathrm{b}}$ & $73.9 \pm 0.76$ & $75.2 \pm 0.55$ & $78.6 \pm 0.28$ & $76.3 \pm 0.58$ & $75.4 \pm 0.36$ & $75.7 \pm 0.15$ & $78.3 \pm 0.59$ & $78.5 \pm 0.87$ \\
\hline Hydrogen & $12.4 \pm 0.08$ & $10.4 \pm 0.16$ & $10.5 \pm 0.07$ & $10.8 \pm 0.05$ & $10.6 \pm 0.08$ & $10.2 \pm 0.11$ & $10.4 \pm 0.11$ & $10.8 \pm 0.07$ & $10.9 \pm 0.03$ \\
\hline Nitrogen & $0.3 \pm 0.01$ & $0.2 \pm 0.01$ & $0.2 \pm 0.01$ & $0.3 \pm 0.01$ & $0.3 \pm 0.02$ & $0.3 \pm 0.01$ & $0.3 \pm 0.01$ & $0.3 \pm 0.01$ & $0.3 \pm 0.01$ \\
\hline Sulfur & $0.4 \pm 0.05$ & $0.3 \pm 0.02$ & $0.6 \pm 0.12$ & $0.6 \pm 0.15$ & $0.6 \pm 0.13$ & $0.7 \pm 0.15$ & $1.0 \pm 0.13$ & $0.7 \pm 0.08$ & $0.9 \pm 0.04$ \\
\hline Oxygen & $0.5 \pm 0.03$ & $3.9 \pm 0.1$ & $4.2 \pm 0.08$ & $4.7 \pm 0.08$ & $4.5 \pm 0.01$ & $5.0 \pm 0.02$ & $5.0 \pm 0.02$ & $4.8 \pm 0.01$ & $4.7 \pm 0.04$ \\
\hline
\end{tabular}

average weight percentage by mass

b. standard deviation 
Table S-3a. Acidic species identified at signal magnitude greater than $6 \sigma$ baseline rms noise in MWO at $m / z 501$ (41 Peaks)

Elemental Composition Measured Mass (Da) Calculated Mass (Da) Mass Error (ppb) m/ $\quad$ m50\% Relative Abundance (\%)

\begin{tabular}{|c|c|c|c|c|c|}
\hline $\mathrm{C}_{37} \mathrm{H}_{26} \mathrm{O}_{2}$ & 501.18605 & 501.186004 & -93 & 1000000 & 0.15 \\
\hline $\mathrm{C}_{36} \mathrm{H}_{27} \mathrm{~N}_{1} \mathrm{O}_{1}{ }^{13} \mathrm{C}_{1}$ & 501.20537 & 501.205343 & -53 & 780000 & 0.26 \\
\hline $\mathrm{C}_{38} \mathrm{H}_{30} \mathrm{O}_{1}$ & 501.22241 & 501.222389 & -42 & 860000 & 0.30 \\
\hline $\mathrm{C}_{35} \mathrm{H}_{34} \mathrm{O}_{1} \mathrm{~S}_{1}$ & 501.22580 & 501.225760 & -79 & 920000 & 0.15 \\
\hline $\mathrm{C}_{37} \mathrm{H}_{30} \mathrm{~N}_{2}$ & 501.23368 & 501.233622 & -115 & 940000 & 0.22 \\
\hline $\mathrm{C}_{37} \mathrm{H}_{31} \mathrm{~N}_{1}{ }^{13} \mathrm{C}_{1}$ & 501.24174 & 501.241729 & -22 & 900000 & 0.57 \\
\hline $\mathrm{C}_{35} \mathrm{H}_{34} \mathrm{O}_{3}$ & 501.24353 & 501.243518 & -23 & 1200000 & 0.23 \\
\hline $\mathrm{C}_{34} \mathrm{H}_{35} \mathrm{~N}_{1} \mathrm{~S}_{1}{ }^{13} \mathrm{C}_{1}$ & 501.24512 & 501.245100 & -40 & 1100000 & 0.24 \\
\hline $\mathrm{C}_{39} \mathrm{H}_{34}$ & 501.25881 & 501.258775 & -71 & 1000000 & 0.48 \\
\hline $\mathrm{C}_{36} \mathrm{H}_{38} \mathrm{~S}_{1}$ & 501.26219 & 501.262146 & -88 & 1000000 & 0.25 \\
\hline $\mathrm{C}_{34} \mathrm{H}_{35} \mathrm{~N}_{1} \mathrm{O}_{2}{ }^{13} \mathrm{C}_{1}$ & 501.26287 & 501.262858 & -23 & 390000 & 0.14 \\
\hline $\mathrm{C}_{36} \mathrm{H}_{38} \mathrm{O}_{2}$ & 501.27992 & 501.279904 & -32 & 990000 & 0.80 \\
\hline $\mathrm{C}_{33} \mathrm{H}_{42} \mathrm{O}_{2} \mathrm{~S}_{1}$ & 501.28337 & 501.283275 & -189 & 870000 & 0.35 \\
\hline $\mathrm{C}_{35} \mathrm{H}_{38} \mathrm{~N}_{2} \mathrm{O}_{1}$ & 501.29116 & 501.291137 & -45 & 940000 & 0.23 \\
\hline $\mathrm{C}_{35} \mathrm{H}_{39} \mathrm{~N}_{1} \mathrm{O}_{1}{ }^{13} \mathrm{C}_{1}$ & 501.29926 & 501.299244 & -32 & 1000000 & 1.15 \\
\hline $\mathrm{C}_{33} \mathrm{H}_{42} \mathrm{O}_{4}$ & 501.30110 & 501.301033 & -133 & 1400000 & 0.13 \\
\hline $\mathrm{C}_{37} \mathrm{H}_{42} \mathrm{O}_{1}$ & 501.31632 & 501.316289 & -61 & 920000 & 1.74 \\
\hline $\mathrm{C}_{34} \mathrm{H}_{46} \mathrm{O}_{1} \mathrm{~S}_{1}$ & 501.31970 & 501.319661 & -78 & 890000 & 0.49 \\
\hline $\mathrm{C}_{32} \mathrm{H}_{43} \mathrm{~N}_{1} \mathrm{O}_{3}{ }^{13} \mathrm{C}_{1}$ & 501.32033 & 501.320373 & 86 & 310000 & 0.14 \\
\hline $\mathrm{C}_{30} \mathrm{H}_{46} \mathrm{O}_{6}$ & 501.32222 & 501.322163 & -114 & 1000000 & 0.14 \\
\hline $\mathrm{C}_{36} \mathrm{H}_{42} \mathrm{~N}_{2}$ & 501.32755 & 501.327523 & -54 & 800000 & 0.45 \\
\hline $\mathrm{C}_{36} \mathrm{H}_{43} \mathrm{~N}_{1}{ }^{13} \mathrm{C}_{1}$ & 501.33564 & 501.335629 & -21 & 960000 & 4.08 \\
\hline $\mathrm{C}_{34} \mathrm{H}_{46} \mathrm{O}_{3}$ & 501.33743 & 501.337419 & -22 & 970000 & 0.34 \\
\hline $\mathrm{C}_{36} \mathrm{H}_{44}{ }^{13} \mathrm{C}_{2}$ & 501.34375 & 501.343736 & -28 & 730000 & 0.15 \\
\hline $\mathrm{C}_{38} \mathrm{H}_{46}$ & 501.35270 & 501.352675 & -50 & 930000 & 2.20 \\
\hline $\mathrm{C}_{33} \mathrm{H}_{47} \mathrm{~N}_{1} \mathrm{O}_{2}{ }^{13} \mathrm{C}_{1}$ & 501.35676 & 501.356759 & -2.6 & 950000 & 0.26 \\
\hline $\mathrm{C}_{31} \mathrm{H}_{50} \mathrm{O}_{5}$ & 501.35861 & 501.358548 & -123 & 1200000 & 0.15 \\
\hline
\end{tabular}




\begin{tabular}{|c|c|c|c|c|c|}
\hline $\mathrm{C}_{35} \mathrm{H}_{50} \mathrm{O}_{2}$ & 501.37382 & 501.373804 & -31 & 940000 & 1.19 \\
\hline $\mathrm{C}_{32} \mathrm{H}_{54} \mathrm{O}_{2} \mathrm{~S}_{1}$ & 501.37721 & 501.377175 & -69 & 1100000 & 0.20 \\
\hline $\mathrm{C}_{34} \mathrm{H}_{51} \mathrm{~N}_{1} \mathrm{O}_{1}{ }^{13} \mathrm{C}_{1}$ & 501.39317 & 501.393144 & -51 & 920000 & 0.54 \\
\hline $\mathrm{C}_{32} \mathrm{H}_{54} \mathrm{O}_{4}$ & 501.39497 & 501.394934 & -72 & 930000 & 0.4 \\
\hline $\mathrm{C}_{29} \mathrm{H}_{58} \mathrm{O}_{4} \mathrm{~S}_{1}$ & 501.39835 & 501.398305 & -90 & 1300000 & 0.12 \\
\hline $\mathrm{C}_{34} \mathrm{H}_{52} \mathrm{O}_{1}{ }^{13} \mathrm{C}_{2}$ & 501.40129 & 501.401251 & -78 & 1100000 & 0.33 \\
\hline $\mathrm{C}_{36} \mathrm{H}_{54} \mathrm{O}_{1}$ & 501.41021 & 501.410190 & -40 & 930000 & 5.11 \\
\hline $\mathrm{C}_{35} \mathrm{H}_{55} \mathrm{~N}_{1}{ }^{13} \mathrm{C}_{1}$ & 501.42955 & 501.429530 & -40 & 1000000 & 4.72 \\
\hline $\mathrm{C}_{33} \mathrm{H}_{58} \mathrm{O}_{3}$ & 501.43134 & 501.431319 & -41 & 1100000 & 0.39 \\
\hline $\mathrm{C}_{35} \mathrm{H}_{56}{ }^{13} \mathrm{C}_{2}$ & 501.43766 & 501.437636 & -47 & 990000 & 0.2 \\
\hline $\mathrm{C}_{37} \mathrm{H}_{58}$ & 501.44661 & 501.446575 & -69 & 920000 & 1.99 \\
\hline $\mathrm{C}_{34} \mathrm{H}_{62} \mathrm{O}_{2}$ & 501.46773 & 501.467705 & -50 & 910000 & 1.00 \\
\hline
\end{tabular}


Table S-3b. Acidic species identified at signal magnitude greater than $6 \sigma$ baseline rms noise in 9 month sample at nominal $\mathrm{m} / \mathrm{z} 501$ (55 peaks)

Elemental Composition Measured Mass (Da) Calculated Mass (Da) Mass Error (ppb) m/Dm50\% Relative Abundance (\%)

\begin{tabular}{|c|c|c|c|c|c|}
\hline $\mathrm{C}_{33} \mathrm{H}_{26} \mathrm{O}_{5}$ & 501.17074 & 501.170747 & 15 & 940000 & 0.2798 \\
\hline $\mathrm{C}_{37} \mathrm{H}_{26} \mathrm{O}_{2}$ & 501.18598 & 501.186004 & 47 & 870000 & 0.1687 \\
\hline $\mathrm{C}_{30} \mathrm{H}_{30} \mathrm{O}_{7}$ & 501.19186 & 501.191877 & 33 & 1300000 & 0.196 \\
\hline $\mathrm{C}_{34} \mathrm{H}_{30} \mathrm{O}_{4}$ & 501.20712 & 501.207133 & 26 & 1000000 & 0.5037 \\
\hline $\mathrm{C}_{31} \mathrm{H}_{34} \mathrm{O}_{4} \mathrm{~S}_{1}$ & 501.21056 & 501.210504 & -110 & 1100000 & 0.2869 \\
\hline $\mathrm{C}_{38} \mathrm{H}_{30} \mathrm{O}_{1}$ & 501.2224 & 501.222389 & -22 & 1200000 & 0.2256 \\
\hline $\mathrm{C}_{33} \mathrm{H}_{31} \mathrm{~N}_{1} \mathrm{O}_{3}{ }^{13} \mathrm{C}_{1}$ & 501.22646 & 501.226473 & 26 & 1100000 & 0.1681 \\
\hline $\mathrm{C}_{31} \mathrm{H}_{34} \mathrm{O}_{6}$ & 501.22826 & 501.228262 & 4.5 & 1100000 & 0.4883 \\
\hline $\mathrm{C}_{28} \mathrm{H}_{38} \mathrm{O}_{6} \mathrm{~S}_{1}$ & 501.23166 & 501.231633 & -53 & 1100000 & 0.1666 \\
\hline $\mathrm{C}_{35} \mathrm{H}_{34} \mathrm{O}_{3}$ & 501.24353 & 501.243518 & -23 & 1000000 & 0.7648 \\
\hline $\mathrm{C}_{32} \mathrm{H}_{38} \mathrm{O}_{3} \mathrm{~S}_{1}$ & 501.2469 & 501.246890 & -21 & 1000000 & 0.561 \\
\hline $\mathrm{C}_{36} \mathrm{H}_{38} \mathrm{~S}_{1}$ & 501.26219 & 501.262146 & -88 & 860000 & 0.1838 \\
\hline $\mathrm{C}_{34} \mathrm{H}_{35} \mathrm{~N}_{1} \mathrm{O}_{2}{ }^{13} \mathrm{C}_{1}$ & 501.26288 & 501.262858 & -43 & 1000000 & 0.248 \\
\hline $\mathrm{C}_{32} \mathrm{H}_{38} \mathrm{O}_{5}$ & 501.26465 & 501.264648 & -4.4 & 1000000 & 1.4376 \\
\hline $\mathrm{C}_{29} \mathrm{H}_{42} \mathrm{O}_{5} \mathrm{~S}_{1}$ & 501.26802 & 501.268019 & -2.2 & 1000000 & 0.4735 \\
\hline $\mathrm{C}_{36} \mathrm{H}_{38} \mathrm{O}_{2}$ & 501.27992 & 501.279904 & -32 & 1200000 & 0.9505 \\
\hline $\mathrm{C}_{33} \mathrm{H}_{42} \mathrm{O}_{2} \mathrm{~S}_{1}$ & 501.28331 & 501.283275 & -70 & 1100000 & 0.7855 \\
\hline $\mathrm{C}_{31} \mathrm{H}_{39} \mathrm{~N}_{1} \mathrm{O}_{4}{ }^{13} \mathrm{C}_{1}$ & 501.28398 & 501.283988 & 15 & 1200000 & 0.298 \\
\hline $\mathrm{C}_{29} \mathrm{H}_{42} \mathrm{O}_{7}$ & 501.28578 & 501.285777 & -5.7 & 970000 & 0.4464 \\
\hline $\mathrm{C}_{31} \mathrm{H}_{40} \mathrm{O}_{4}{ }^{13} \mathrm{C}_{2}$ & 501.29209 & 501.292094 & 8.4 & 1000000 & 0.2211 \\
\hline $\mathrm{C}_{35} \mathrm{H}_{39} \mathrm{~N}_{1} \mathrm{O}_{1}{ }^{13} \mathrm{C}_{1}$ & 501.29923 & 501.299244 & 28 & 1100000 & 0.3392 \\
\hline $\mathrm{C}_{33} \mathrm{H}_{42} \mathrm{O}_{4}$ & 501.30103 & 501.301033 & 6.6 & 980000 & 3.6678 \\
\hline $\mathrm{C}_{30} \mathrm{H}_{46} \mathrm{O}_{4} \mathrm{~S}_{1}$ & 501.30442 & 501.304404 & -31 & 1100000 & 1.0768 \\
\hline $\mathrm{C}_{37} \mathrm{H}_{42} \mathrm{O}_{1}$ & 501.31627 & 501.316289 & 39 & 1000000 & 0.7745 \\
\hline $\mathrm{C}_{34} \mathrm{H}_{46} \mathrm{O}_{1} \mathrm{~S}_{1}$ & 501.31969 & 501.319661 & -59 & 1200000 & 0.2608 \\
\hline $\mathrm{C}_{32} \mathrm{H}_{43} \mathrm{~N}_{1} \mathrm{O}_{3}{ }^{13} \mathrm{C}_{1}$ & 501.32036 & 501.320373 & 26 & 1200000 & 0.6355 \\
\hline
\end{tabular}




\begin{tabular}{|c|c|c|c|c|c|}
\hline $\mathrm{C}_{30} \mathrm{H}_{46} \mathrm{O}_{6}$ & 501.32216 & 501.322163 & 5.3 & 1100000 & 1.5782 \\
\hline $\mathrm{C}_{32} \mathrm{H}_{44} \mathrm{O}_{3}{ }^{13} \mathrm{C}_{2}$ & 501.32848 & 501.328480 & -0.59 & 1100000 & 0.2608 \\
\hline $\mathrm{C}_{36} \mathrm{H}_{43} \mathrm{~N}_{1}{ }^{13} \mathrm{C}_{1}$ & 501.3356 & 501.335629 & 59 & 910000 & 0.412 \\
\hline $\mathrm{C}_{34} \mathrm{H}_{46} \mathrm{O}_{3}$ & 501.33742 & 501.337419 & -2.4 & 1100000 & 6.0541 \\
\hline $\mathrm{C}_{31} \mathrm{H}_{50} \mathrm{O}_{3} \mathrm{~S}_{1}$ & 501.3408 & 501.340790 & -20 & 1000000 & 1.2012 \\
\hline $\mathrm{C}_{29} \mathrm{H}_{48} \mathrm{O}_{5}{ }^{13} \mathrm{C}_{2}$ & 501.3496 & 501.349609 & 18 & 930000 & 0.2329 \\
\hline $\mathrm{C}_{38} \mathrm{H}_{46}$ & 501.35265 & 501.352675 & 50 & 1000000 & 0.8985 \\
\hline $\mathrm{C}_{33} \mathrm{H}_{47} \mathrm{~N}_{1} \mathrm{O}_{2}{ }^{13} \mathrm{C}_{1}$ & 501.35676 & 501.356759 & -2.6 & 960000 & 0.8585 \\
\hline $\mathrm{C}_{31} \mathrm{H}_{50} \mathrm{O}_{5}$ & 501.35854 & 501.358548 & 16 & 1000000 & 3.8003 \\
\hline $\mathrm{C}_{28} \mathrm{H}_{54} \mathrm{O}_{5} \mathrm{~S}_{1}$ & 501.36192 & 501.361919 & -1.4 & 1000000 & 0.6467 \\
\hline $\mathrm{C}_{35} \mathrm{H}_{50} \mathrm{O}_{2}$ & 501.37381 & 501.373804 & -11 & 1100000 & 5.8216 \\
\hline $\mathrm{C}_{32} \mathrm{H}_{54} \mathrm{O}_{2} \mathrm{~S}_{1}$ & 501.37718 & 501.377175 & -9.1 & 970000 & 0.5446 \\
\hline $\mathrm{C}_{30} \mathrm{H}_{51} \mathrm{~N}_{1} \mathrm{O}_{4}{ }^{13} \mathrm{C}_{1}$ & 501.37791 & 501.377888 & -44 & 1100000 & 0.197 \\
\hline $\mathrm{C}_{30} \mathrm{H}_{52} \mathrm{O}_{4}{ }^{13} \mathrm{C}_{2}$ & 501.386 & 501.385995 & -11 & 990000 & 0.4897 \\
\hline $\mathrm{C}_{34} \mathrm{H}_{51} \mathrm{~N}_{1} \mathrm{O}_{1}{ }^{13} \mathrm{C}_{1}$ & 501.39316 & 501.393144 & -31 & 950000 & 0.9392 \\
\hline $\mathrm{C}_{32} \mathrm{H}_{54} \mathrm{O}_{4}$ & 501.39493 & 501.394934 & 7.3 & 1000000 & 9.0264 \\
\hline $\mathrm{C}_{29} \mathrm{H}_{58} \mathrm{O}_{4} \mathrm{~S}_{1}$ & 501.3983 & 501.398305 & -9.1 & 1000000 & 4.4713 \\
\hline $\mathrm{C}_{36} \mathrm{H}_{54} \mathrm{O}_{1}$ & 501.41019 & 501.410190 & -0.36 & 1000000 & 2.802 \\
\hline $\mathrm{C}_{33} \mathrm{H}_{58} \mathrm{O}_{1} \mathrm{~S}_{1}$ & 501.41364 & 501.413561 & -160 & 1300000 & 0.2111 \\
\hline $\mathrm{C}_{31} \mathrm{H}_{55} \mathrm{~N}_{1} \mathrm{O}_{3}{ }^{13} \mathrm{C}_{1}$ & 501.41432 & 501.414274 & -93 & 1400000 & 0.39 \\
\hline $\mathrm{C}_{31} \mathrm{H}_{56} \mathrm{O}_{3}{ }^{13} \mathrm{C}_{2}$ & 501.42237 & 501.422380 & 20 & 1000000 & 0.8533 \\
\hline $\mathrm{C}_{35} \mathrm{H}_{55} \mathrm{~N}_{1}{ }^{13} \mathrm{C}_{1}$ & 501.42953 & 501.429530 & -0.56 & 990000 & 0.8595 \\
\hline $\mathrm{C}_{33} \mathrm{H}_{58} \mathrm{O}_{3}$ & 501.43133 & 501.431319 & -22 & 1000000 & 9.2402 \\
\hline $\mathrm{C}_{30} \mathrm{H}_{62} \mathrm{O}_{3} \mathrm{~S}_{1}$ & 501.43471 & 501.434690 & -39 & 790000 & 0.408 \\
\hline $\mathrm{C}_{35} \mathrm{H}_{56}{ }^{13} \mathrm{C}_{2}$ & 501.43758 & 501.437636 & 110 & 870000 & 0.1587 \\
\hline $\mathrm{C}_{37} \mathrm{H}_{58}$ & 501.44658 & 501.446575 & -9.3 & 1000000 & 1.8152 \\
\hline $\mathrm{C}_{32} \mathrm{H}_{59} \mathrm{~N}_{1} \mathrm{O}_{2}{ }^{13} \mathrm{C}_{1}$ & 501.45069 & 501.450659 & -62 & 990000 & 0.1737 \\
\hline $\mathrm{C}_{34} \mathrm{H}_{62} \mathrm{O}_{2}$ & 501.46772 & 501.467705 & -31 & 1100000 & 12.7426 \\
\hline $\mathrm{C}_{35} \mathrm{H}_{66} \mathrm{O}_{1}$ & 501.5041 & 501.504090 & -20 & 980000 & 1.5078 \\
\hline
\end{tabular}


Table S-3c. Acidic species identified at signal magnitude greater than $6 \sigma$ baseline rms noise in $36 \mathrm{month}$ sample at nominal $\mathrm{m} / \mathrm{z} 501$ (47 peaks)

\section{Elemental Composition Measured Mass (Da) Calculated Mass (Da) Mass Error (ppb) m/Dm50\% Relative Abundance (\%)}

\begin{tabular}{|c|c|c|c|c|c|}
\hline $\mathrm{C}_{33} \mathrm{H}_{26} \mathrm{O}_{5}$ & 501.17075 & 501.170747 & -5.2 & 1100000 & 0.164 \\
\hline $\mathrm{C}_{34} \mathrm{H}_{30} \mathrm{O}_{4}$ & 501.20713 & 501.207133 & 5.8 & 1000000 & 0.3284 \\
\hline $\mathrm{C}_{31} \mathrm{H}_{34} \mathrm{O}_{4} \mathrm{~S}_{1}$ & 501.21048 & 501.210504 & 48 & 720000 & 0.2127 \\
\hline $\mathrm{C}_{31} \mathrm{H}_{34} \mathrm{O}_{6}$ & 501.2283 & 501.228262 & -75 & 1300000 & 0.1869 \\
\hline $\mathrm{C}_{35} \mathrm{H}_{34} \mathrm{O}_{3}$ & 501.24354 & 501.243518 & -43 & 910000 & 0.4306 \\
\hline $\mathrm{C}_{32} \mathrm{H}_{38} \mathrm{O}_{3} \mathrm{~S}_{1}$ & 501.24692 & 501.246890 & -61 & 1200000 & 0.32 \\
\hline $\mathrm{C}_{32} \mathrm{H}_{38} \mathrm{O}_{5}$ & 501.26463 & 501.264648 & 35 & 890000 & 0.8428 \\
\hline $\mathrm{C}_{29} \mathrm{H}_{42} \mathrm{O}_{5} \mathrm{~S}_{1}$ & 501.26805 & 501.268019 & -62 & 810000 & 0.2993 \\
\hline $\mathrm{C}_{36} \mathrm{H}_{38} \mathrm{O}_{2}$ & 501.27992 & 501.279904 & -32 & 1100000 & 0.4609 \\
\hline $\mathrm{C}_{33} \mathrm{H}_{42} \mathrm{O}_{2} \mathrm{~S}_{1}$ & 501.28329 & 501.283275 & -30 & 1000000 & 0.5482 \\
\hline $\mathrm{C}_{31} \mathrm{H}_{39} \mathrm{~N}_{1} \mathrm{O}_{4}{ }^{13} \mathrm{C}_{1}$ & 501.284 & 501.283988 & -25 & 1400000 & 0.1684 \\
\hline $\mathrm{C}_{29} \mathrm{H}_{42} \mathrm{O}_{7}$ & 501.28577 & 501.285777 & 14 & 1000000 & 0.2678 \\
\hline $\mathrm{C}_{33} \mathrm{H}_{42} \mathrm{O}_{4}$ & 501.30104 & 501.301033 & -13 & 940000 & 2.2118 \\
\hline $\mathrm{C}_{30} \mathrm{H}_{46} \mathrm{O}_{4} \mathrm{~S}_{1}$ & 501.30442 & 501.304404 & -31 & 870000 & 0.7414 \\
\hline $\mathrm{C}_{37} \mathrm{H}_{42} \mathrm{O}_{1}$ & 501.31631 & 501.316289 & -41 & 830000 & 0.2058 \\
\hline $\mathrm{C}_{34} \mathrm{H}_{46} \mathrm{O}_{1} \mathrm{~S}_{1}$ & 501.31971 & 501.319661 & -99 & 970000 & 0.181 \\
\hline $\mathrm{C}_{32} \mathrm{H}_{43} \mathrm{~N}_{1} \mathrm{O}_{3}{ }^{13} \mathrm{C}_{1}$ & 501.32035 & 501.320373 & 46 & 1200000 & 0.4133 \\
\hline $\mathrm{C}_{30} \mathrm{H}_{46} \mathrm{O}_{6}$ & 501.32216 & 501.322163 & 5.3 & 1100000 & 1.0112 \\
\hline $\mathrm{C}_{32} \mathrm{H}_{44} \mathrm{O}_{3}{ }^{13} \mathrm{C}_{2}$ & 501.32848 & 501.328480 & -0.59 & 1200000 & 0.2102 \\
\hline $\mathrm{C}_{34} \mathrm{H}_{46} \mathrm{O}_{3}$ & 501.33743 & 501.337419 & -22 & 990000 & 4.2377 \\
\hline $\mathrm{C}_{31} \mathrm{H}_{50} \mathrm{O}_{3} \mathrm{~S}_{1}$ & 501.3408 & 501.340790 & -20 & 1000000 & 0.792 \\
\hline $\mathrm{C}_{33} \mathrm{H}_{47} \mathrm{~N}_{1} \mathrm{O}_{2}{ }^{13} \mathrm{C}_{1}$ & 501.35676 & 501.356759 & -2.6 & 1200000 & 0.41 \\
\hline $\mathrm{C}_{31} \mathrm{H}_{50} \mathrm{O}_{5}$ & 501.35854 & 501.358548 & 16 & 1000000 & 2.5749 \\
\hline $\mathrm{C}_{28} \mathrm{H}_{54} \mathrm{O}_{5} \mathrm{~S}_{1}$ & 501.36193 & 501.361919 & -21 & 820000 & 0.394 \\
\hline
\end{tabular}




$\begin{array}{llllll}\mathrm{C}_{33} \mathrm{H}_{48} \mathrm{O}_{2}{ }^{13} \mathrm{C}_{2} & 501.36481 & 501.364865 & 110 & 1200000 & 0.2728 \\ \mathrm{C}_{35} \mathrm{H}_{50} \mathrm{O}_{2} & 501.37381 & 501.373804 & -11 & 1000000 & 4.834 \\ \mathrm{C}_{32} \mathrm{H}_{54} \mathrm{O}_{2} \mathrm{~S}_{1} & 501.37717 & 501.377175 & 11 & 1000000 & 0.3463 \\ \mathrm{C}_{30} \mathrm{H}_{52} \mathrm{O}_{4}{ }^{13} \mathrm{C}_{2} & 501.386 & 501.385995 & -11 & 1100000 & 0.405 \\ \mathrm{C}_{34} \mathrm{H}_{51} \mathrm{~N}_{1} \mathrm{O}_{1}{ }^{13} \mathrm{C}_{1} & 501.39316 & 501.393144 & -31 & 850000 & 0.4304 \\ \mathrm{C}_{32} \mathrm{H}_{54} \mathrm{O}_{4} & 501.39494 & 501.394934 & -13 & 1000000 & 9.3933 \\ \mathrm{C}_{29} \mathrm{H}_{58} \mathrm{O}_{4} \mathrm{~S}_{1} & 501.39831 & 501.398305 & -10 & 980000 & 2.7398 \\ \mathrm{C}_{36} \mathrm{H}_{54} \mathrm{O}_{1} & 501.41018 & 501.410190 & 20 & 970000 & 1.328 \\ \mathrm{C}_{33} \mathrm{H}_{58} \mathrm{O}_{1} \mathrm{~S}_{1} & 501.41357 & 501.413561 & -18 & 640000 & 0.1798 \\ \mathrm{C}_{31} \mathrm{H}_{56} \mathrm{O}_{3}{ }^{13} \mathrm{C}_{2} & 501.42238 & 501.422380 & 0.18 & 1000000 & 0.7842 \\ \mathrm{C}_{33} \mathrm{H}_{58} \mathrm{O}_{3} & 501.43133 & 501.431319 & -22 & 970000 & 12.3926 \\ \mathrm{C}_{30} \mathrm{H}_{62} \mathrm{O}_{3} \mathrm{~S}_{1} & 501.4347 & 501.434690 & -19 & 980000 & 0.3906 \\ \mathrm{C}_{37} \mathrm{H}_{58} & 501.44657 & 501.446575 & 11 & 1200000 & 0.1638 \\ \mathrm{C}_{32} \mathrm{H}_{60} \mathrm{O}_{2}{ }^{13} \mathrm{C}_{2} & 501.45877 & 501.458766 & -8.8 & 1000000 & 1.8316 \\ \mathrm{C}_{29} \mathrm{H}_{62} \mathrm{~N}_{2} \mathrm{O}_{4} & 501.46364 & 501.463682 & 84 & 480000 & 0.5702 \\ \mathrm{C}_{34} \mathrm{H}_{62} \mathrm{O}_{2} & 501.46772 & 501.467705 & -31 & 1000000 & 39.9451\end{array}$


Table S-3d. Acidic species identified at signal magnitude greater than $6 \sigma$ baseline rms noise in 48 month sample at nominal $\mathrm{m} / \mathrm{z} 501$ (15 peaks)

\begin{tabular}{|c|c|c|c|c|c|}
\hline Elemental Composition & Measured Mass (Da) & Calculated Mass (Da) & Mass Error (ppb) & $\mathrm{m} / \Delta \mathrm{m} 50 \%$ & Relative Abundance (\%) \\
\hline $\mathrm{C}_{33} \mathrm{H}_{42} \mathrm{O}_{4}$ & 501.30103 & 501.301033 & 6.6 & 1300000 & 0.2601 \\
\hline $\mathrm{C}_{30} \mathrm{H}_{46} \mathrm{O}_{6}$ & 501.32216 & 501.322162 & 5.3 & 1200000 & 0.401 \\
\hline $\mathrm{C}_{34} \mathrm{H}_{46} \mathrm{O}_{3}$ & 501.33742 & 501.337418 & -2.4 & 1300000 & 0.6457 \\
\hline $\mathrm{C}_{31} \mathrm{H}_{50} \mathrm{O}_{5}$ & 501.35854 & 501.358548 & 16 & 1300000 & 1.03 \\
\hline $\mathrm{C}_{35} \mathrm{H}_{50} \mathrm{O}_{2}$ & 501.37381 & 501.373804 & -11 & 1300000 & 1.1434 \\
\hline $\mathrm{C}_{32} \mathrm{H}_{54} \mathrm{O}_{4}$ & 501.39493 & 501.394933 & 7.3 & 1200000 & 19.1036 \\
\hline $\mathrm{C}_{29} \mathrm{H}_{58} \mathrm{O}_{4} \mathrm{~S}_{1}$ & 501.39831 & 501.398304 & -10.4 & 940000 & 0.232 \\
\hline $\mathrm{C}_{29} \mathrm{H}_{58} \mathrm{O}_{6}$ & 501.41609 & 501.416063 & -53.8 & 1300000 & 0.389 \\
\hline $\mathrm{C}_{31} \mathrm{H}_{56} \mathrm{O}_{3}{ }^{13} \mathrm{C}_{2}$ & 501.42234 & 501.422380 & 80 & 1200000 & 0.2535 \\
\hline $\mathrm{C}_{33} \mathrm{H}_{58} \mathrm{O}_{3}$ & 501.43132 & 501.431319 & -1.6 & 1200000 & 4.0731 \\
\hline $\mathrm{C}_{32} \mathrm{H}_{60} \mathrm{O}_{2}{ }^{13} \mathrm{C}_{2}$ & 501.45877 & 501.458765 & -8.8 & 1200000 & 0.7071 \\
\hline $\mathrm{C}_{34} \mathrm{H}_{62} \mathrm{O}_{2}$ & 501.4677 & 501.467704 & 9.4 & 1300000 & 9.9998 \\
\hline
\end{tabular}

